Case Report

\title{
Primary Cutaneous Actinomycosis along with the Surgical Scar on the Hand
}

\author{
Reza M. Robati, ${ }^{1}$ Nasim Niknezhad, ${ }^{1}$ \\ Farahnaz Bidari-Zerehpoush, ${ }^{2}$ and Nakisa Niknezhad ${ }^{1}$ \\ ${ }^{1}$ Skin Research Center, Shahid Beheshti University of Medical Sciences, Tehran, Iran \\ ${ }^{2}$ Department of Pathology, Loghman-Hakim Hospital, Shahid Beheshti University of Medical Sciences, Tehran, Iran \\ Correspondence should be addressed to Reza M. Robati; rezarobati@sbmu.ac.ir
}

Received 19 July 2016; Accepted 25 October 2016

Academic Editor: Larry M. Bush

Copyright (C) 2016 Reza M. Robati et al. This is an open access article distributed under the Creative Commons Attribution License, which permits unrestricted use, distribution, and reproduction in any medium, provided the original work is properly cited.

Primary cutaneous actinomycosis is a rare clinical form with variable presentation. The tumoral presentation of actinomycosis as pseudocarcinomatous or sarcomatous masses is completely rare. The management of cutaneous actinomycosis needs proper antibiotic treatment and surgical resection would not be adequate alone. Herein, we report a case of primary cutaneous actinomycosis on the dorsal surface of the hand as draining and infiltrated lesions along with the scar of previous tumor excision that had not received proper antibiotics after the surgery.

\section{Introduction}

Actinomycosis is a slowly progressive infection caused by anaerobic or microaerophilic bacteria, primarily of the genus Actinomyces, and normally colonizes the mouth, colon, and urogenital tract. It has no predilection for age, race, season, or occupation and is seen commonly in tropical countries. The previous male preponderance is now surpassed by an increasing number of females being infected, as it is associated with long-term use of intrauterine devices [1,2].

Actinomycosis is characterized by chronic and progressive suppurative inflammation, commonly seen on the neck, thorax, and abdomen. Five main clinical types are cervicofacial $(60 \%)$, thoracic $(20 \%)$, abdominal (15\%), pelvic, and primary cutaneous, the last being rare and having a variable manifestation [2]. It can manifest as subcutaneous nodules that extend slowly. The classical presentation includes a chronic, localized infiltrative process with abscess, fistula formation, and draining sinuses. Tumoral presentation as pseudocarcinomatous or sarcomatous masses is very rare. The physician should consider actinomycosis on the differential diagnosis list of any patients with chronic nonhealing lesions on the face or extremities [3]. Herein, we report a case of primary cutaneous actinomycosis on the dorsal surface of the hand as draining and infiltrated lesions along with the scar of previous tumor excision.

\section{Case Report}

A 35-year-old man has been referred to our Dermatology Clinic with the complaint of painful firm immobile nodules along with a surgical scar on the dorsum of his hand. There were also pus drainage and ulceration in some part of the scar. He claimed that there was a tumoral swelling at the dorsum of his left hand in the second metacarpal area for 12 months. It had been gradually increased in size and led to the restriction of the movement of the index finger. Therefore, he came to an orthopedic surgeon and underwent a tumor excision operation about six months ago. He completely improved after this surgery and did not follow up any further treatment. Four months after the surgery, some painful immobile ulcerated nodules with low discharge appeared on the way of the surgical scar. He works as an electrical technician but there was no obvious history of trauma or significant medical problem. Physical examination showed swollen areas at the dorsum of the left hand and palpable tender immobile nodules on the surgical scar with low 


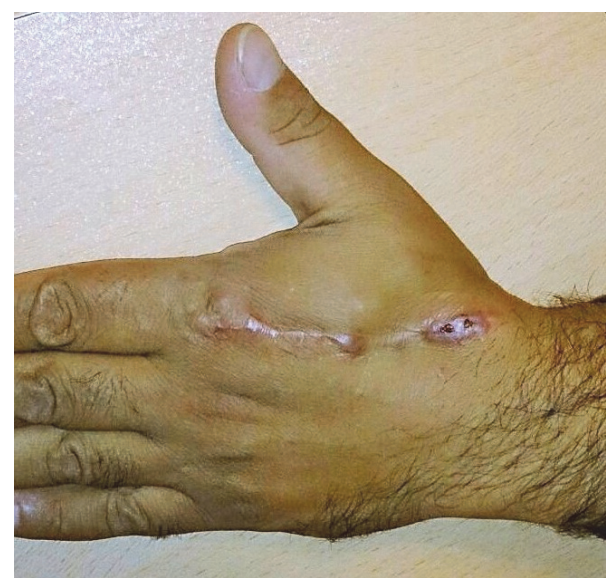

FIGURE 1: Swollen areas at the dorsum of the left hand and tender immobile nodules on the surgical scar.

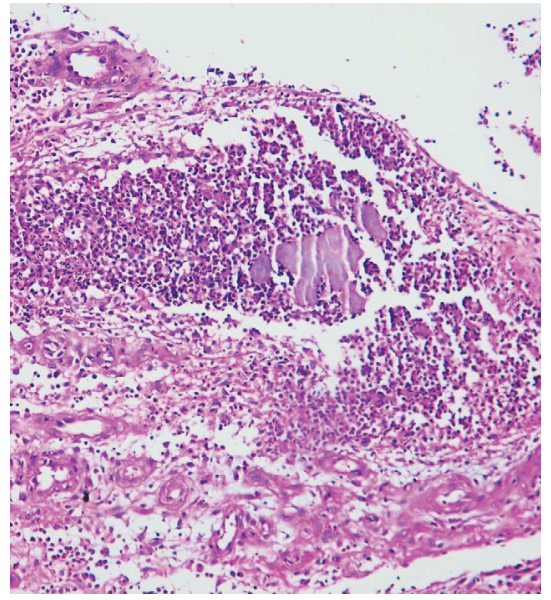

FIGURE 2: Histopathology view: ulcerated epidermis with underlying neutrophilic microabscess surrounded by granulation tissues consisting of plasma cell, macrophage, and fibroblast infiltration $(\mathrm{H} \& \mathrm{E} * 10)$.

discharge. Some nodules showed ulcerated crusted surface (Figure 1).

The hematological and biochemical laboratory tests were normal. The hand radiography was done and reported as normal. Therefore, he underwent an incisional biopsy of the nodules. Microscopic examination reveals an ulcerated epidermis with underlying neutrophilic microabscesses surrounded by granulation tissues consisting of plasma cell, macrophage, and fibroblast infiltration. Sulfur granules were found at the center of these inflammatory reactions as filamentous basophilic radiating fungal-like structures in the dermis (Figures 2 and 3).

Regarding these clinical and histopathological data, the diagnosis of actinomycosis was made. Moreover, we asked the patient to bring us any histopathology report of the tumor excised on his hand about one year before his admission. Surprisingly, the diagnosis of actinomycosis had also been mentioned in that report. But he had not come to his

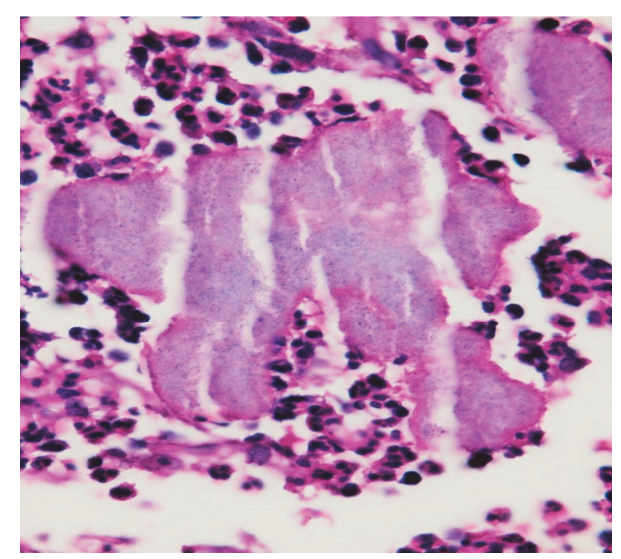

FIGURE 3: Sulfur granules were found at the center of the inflammatory reactions as filamentous basophilic radiating fungal-like structures in the dermis $(\mathrm{H} \& \mathrm{E} * 40)$.

surgeon for any further treatment and the recurrence of the lesions occurred on the surgical scar. The patient was treated with intravenous penicillin $G$ for four weeks and then oral penicillin. Resolution of symptoms and sings occurred but the patient did not come back for further follow-up.

\section{Discussion}

Actinomycosis is believed to be acquired by endogenous implantation into deep tissues where anaerobic environments exist. Perforating wounds, dental surgery, or compound fractures are some of the routes of infection. Primary actinomycosis of the extremity is rare because of the endogenous habitat of the etiologic organism. Cutaneous localization of actinomycosis generally occurs by contiguity of primary centers by direct inoculation or by extent through the bloodstream. In the latter case, there are often several lesions. Posttraumatic actinomycosis and cases associated with insect bites have also been reported $[4,5]$. There are limited reports of primary cutaneous actinomycosis with different locations of involvement such as scalp, gluteal area, hand, axilla, or chest wall [3-8]. Minor trauma due to thick grass, thorn prick, or contact with human feces might also be associated [2]. However, several cases show no obvious history of trauma, occupational contact, or any exogenous focus of infection $[2,5,7]$. Our patient also mentioned no history of obvious trauma but, regarding his job as an electrical technician, he could have experienced some unnoticed traumas on his hand to be inoculated with Actinomyces spp.

Cutaneous actinomycosis needs to be differentiated from other chronic skin diseases, such as cutaneous tuberculosis, sporotrichosis, nocardiosis, and skin and soft tissue tumors or malignancy. Diagnosis is based on identification of sulfur granules in histological examination and also by a positive culture. The histological picture consist of suppurative inflammation with abscesses and pus filled sinus tracts in which bacteria form typical granular colonies (sulfur granules) composed of radiating Gram-positive filaments [4, 6]. The sulfur granules were described as basophilic masses with 
eosinophilic terminal clubs on staining with hematoxylin and eosin. These findings are highly suggestive of the diagnosis but are not specific, as they can be encountered in other pathogenic conditions such as nocardiosis. However, Gram staining usually shows Gram-positive filamentous branching bacteria at the periphery of the granule that is highly suggestive of actinomycosis [9]. Moreover, Nocardia can usually be differentiated from Actinomyces by acidfast staining, as Nocardia characteristically display varying degrees of acid fastness on Ziehl-Neelsen staining due to the mycolic acid content of the cell wall. Another valuable clue is that Nocardia grow under aerobic environments, whereas Actinomyces grow under anaerobic settings [10]. We could not perform the culture due to our limited facilities but the characteristic sulfur granules were obvious on the histopathology examination.

The management of cutaneous actinomycosis is high dose intravenous antibiotics for 4-6 weeks followed by oral antibiotics for 6-12 months. Beta-lactams including penicillin $\mathrm{G}$ are the treatment of choice. In penicillin resistant cases, we could use the other antibiotics such as clindamycin, erythromycin, tetracycline, and chloramphenicol [3, 11]. Amoxicillin/clavulanate could be another suitable option for the treatment of cutaneous actinomycosis. In a recent study, these drugs have been administered for the treatment of cutaneous actinomycosis as suitable oral agents for maximum of 12 weeks with considerable efficacy [12]. Surgery alone is not curative and combination of surgical intervention with antibiotics leads to faster resolution of lesion with cosmetically better results and decreases the rate of recurrence $[3,11]$. Our patient had not received any antibiotic therapy after the excisional surgery of the actinomycosis lesion on his hand. Therefore, he faced the recurrence of disease along with the surgical scar after some months.

In conclusion, our reported case seems to be a bit distinctive due to its location on the hand as a rare location of primary cutaneous actinomycosis [6] and especially the recurrence of the lesions along with the surgical scar of the primary lesion excision. Therefore, it is recommended to keep in mind actinomycosis in differential diagnosis of any nodular, draining, or recurrent lesions on the extremities. We also put emphasis on the necessity of prolonged antibiotic therapy when the diagnosis of actinomycosis is made as the excisional surgery would not be adequate alone.

\section{Competing Interests}

The authors declare that they have no competing interests.

\section{References}

[1] G. Pulverer, H. Schütt-Gerowitt, and K. P. Schaal, "Human cervicofacial actinomycoses: microbiological data for 1997 cases," Clinical Infectious Diseases, vol. 37, no. 4, pp. 490-497, 2003.

[2] N. Jivani and P. Nair, "Primary cutaneous actinomycosis over right gluteal region," Indian Dermatology Online Journal, vol. 7, no. 3, pp. 217-219, 2016.

[3] M. Akhtar, M. P. Zade, P. L. Shahane, A. P. Bangde, and S. M. Soitkar, "Scalp actinomycosis presenting as soft tissue tumour: a case report with literature review," International Journal of Surgery Case Reports, vol. 16, pp. 99-101, 2015.

[4] M. S. Fazeli and H. Bateni, "Actinomycosis: a rare soft tissue infection," Dermatology Online Journal, vol. 11, no. 3, article 18, 2005.

[5] D. Roy, P. G. Roy, and P. K. Misra, "An interesting case of primary cutaneous actinomycosis," Dermatology Online Journal, vol. 9, no. 5, article 17, 2003.

[6] C. Aypak, H. Gökçe, A. Altunsoy, S. Koç, and S. Kaplan, "Primary actinomycosis of hand: a rare soft tissue infection," Journal of Dermatology, vol. 39, no. 8, pp. 741-742, 2012.

[7] M. Bose, R. Ghosh, K. Mukherjee, and L. Ghoshal, "Primary cutaneous actinomycosis: a case report," Journal of Clinical and Diagnostic Research, vol. 8, no. 7, pp. 3-5, 2014.

[8] V. Mehta and C. Balachandran, "Primary cutaneous actinomycosis on the chest wall," Dermatology Online Journal, vol. 14, no. 8 , article 13, 2008.

[9] F. Valour, A. Sénéchal, C. Dupieux et al., "Actinomycosis: etiology, clinical features, diagnosis, treatment, and management," Infection and Drug Resistance, vol. 7, pp. 183-197, 2014.

[10] B. L. Beaman and L. Beaman, "Nocardia species: host-parasite relationships," Clinical Microbiology Reviews, vol. 7, no. 2, pp. 213-264, 1994.

[11] C. Steininger and B. Willinger, "Resistance patterns in clinical isolates of pathogenic Actinomyces species," Journal of Antimicrobial Chemotherapy, vol. 71, no. 2, pp. 422-427, 2016.

[12] A. Bonifaz, A. Tirado-Sánchez, L. Calderón et al., "Treatment of cutaneous actinomycosis with amoxicillin/clavulanic acid," Journal of Dermatological Treatment, 2016. 


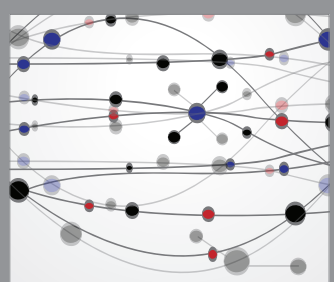

The Scientific World Journal
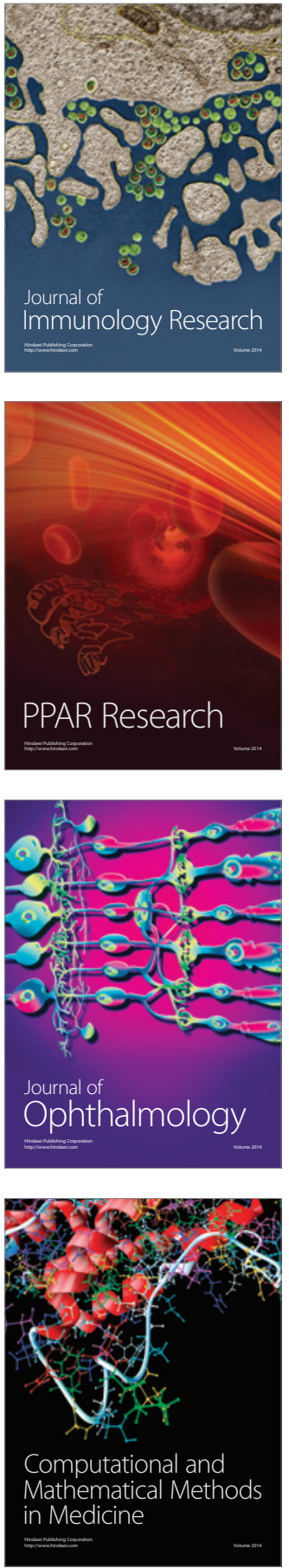

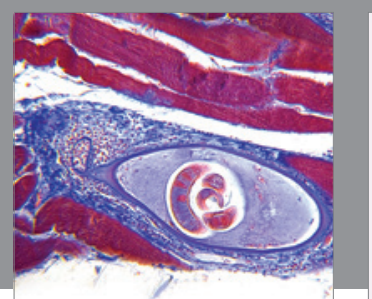

Gastroenterology Research and Practice

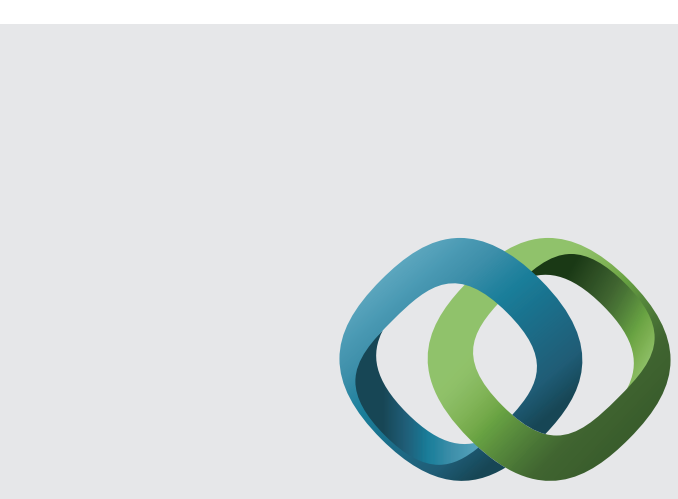

\section{Hindawi}

Submit your manuscripts at

http://www.hindawi.com
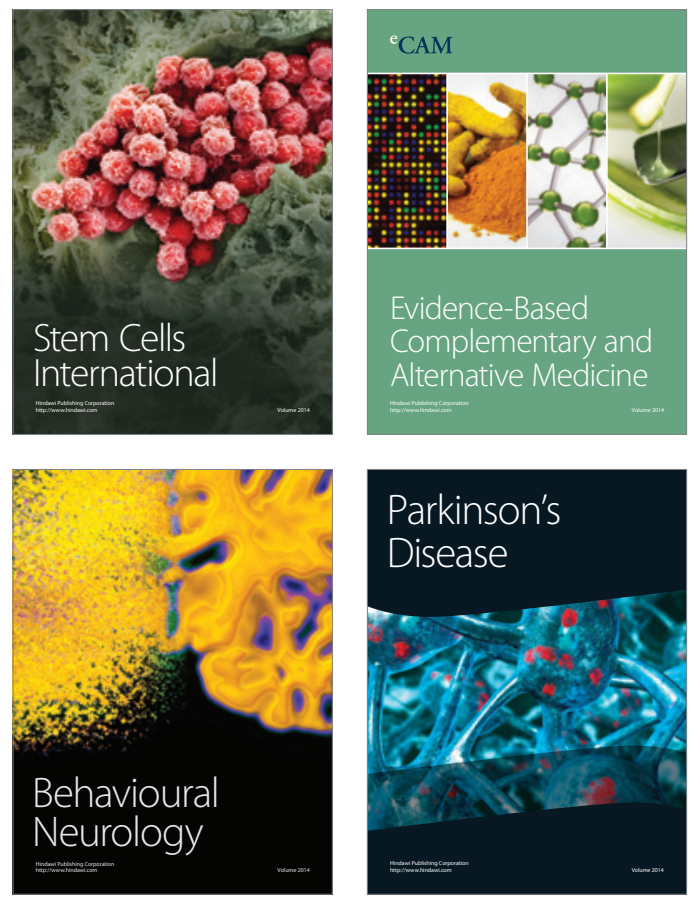
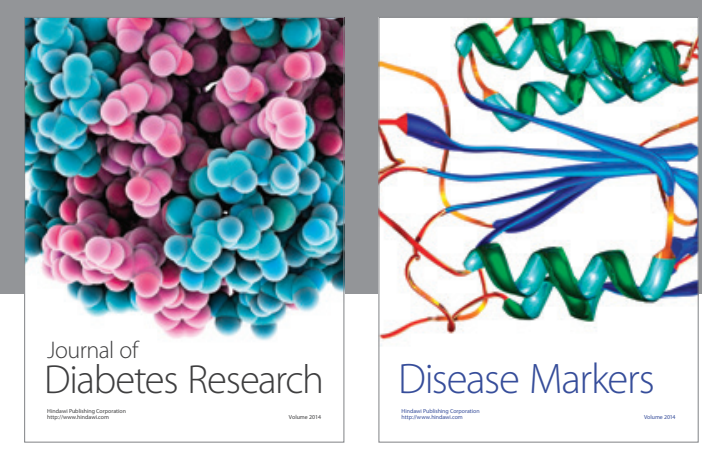

Disease Markers
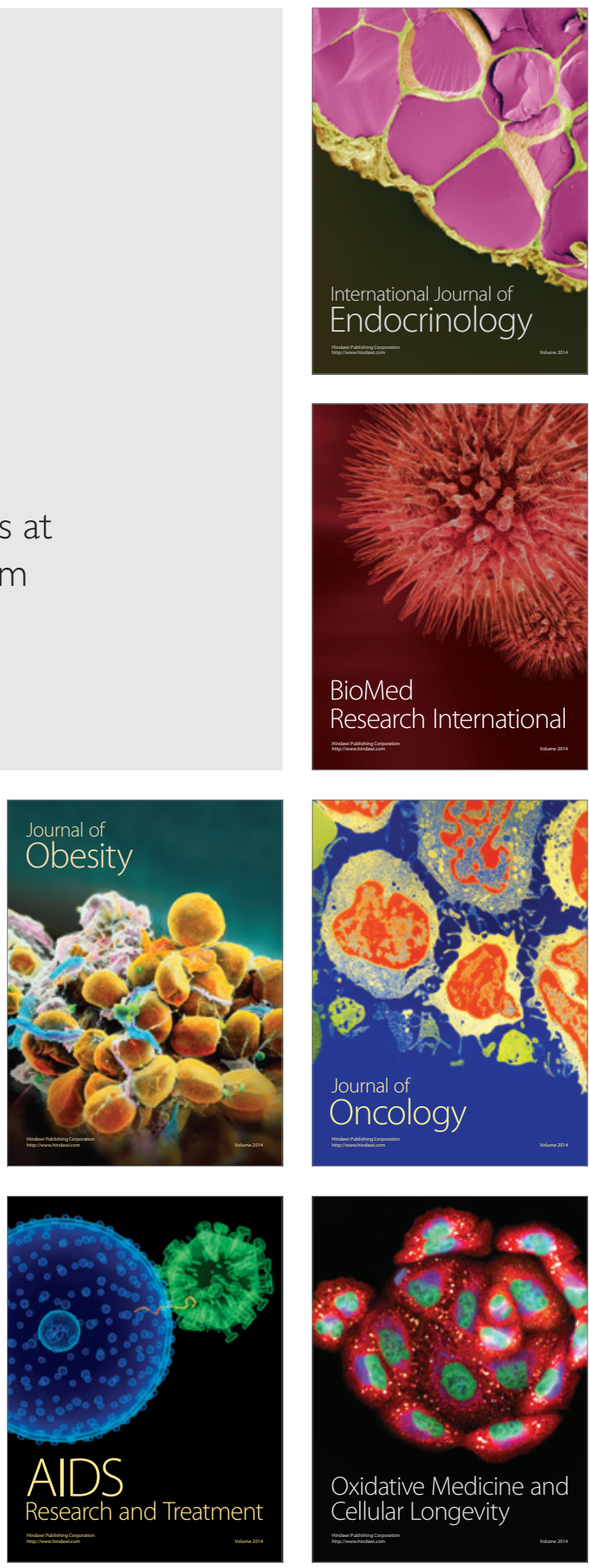